\title{
Collisional quenching of highly rotationally excited $\mathrm{HF}^{\star}$
}

\author{
B. Yang ${ }^{1}$, K. M. Walker ${ }^{1}$, R. C. Forrey ${ }^{2}$, P. C. Stancil ${ }^{1}$, and N. Balakrishnan ${ }^{3}$ \\ ${ }^{1}$ Department of Physics and Astronomy and the Center for Simulational Physics, The University of Georgia, Athens, GA 30602, \\ USA \\ e-mail: yang@physast.uga.edu \\ 2 Department of Physics, Penn State University, Berks Campus, Reading, PA 19610, USA \\ 3 Department of Chemistry, University of Nevada, Las Vegas, NV 89154, USA \\ Received 3 February 2015 / Accepted 23 April 2015
}

\section{ABSTRACT}

\begin{abstract}
Context. Collisional excitation rate coefficients play an important role in the dynamics of energy transfer in the interstellar medium. In particular, accurate rotational excitation rates are needed to interpret microwave and infrared observations of the interstellar gas for nonlocal thermodynamic equilibrium line formation.

Aims. Theoretical cross sections and rate coefficients for collisional deexcitation of rotationally excited HF in the vibrational ground state are reported.

Methods. The quantum-mechanical close-coupling approach implemented in the nonreactive scattering code MOLSCAT was applied in the cross section and rate coefficient calculations on an accurate 2D HF-He potential energy surface. Estimates of rate coefficients for $\mathrm{H}$ and $\mathrm{H}_{2}$ colliders were obtained from the HF-He collisional data with a reduced-potential scaling approach.

Results. The calculation of state-to-state rotational quenching cross sections for HF due to He with initial rotational levels up to $j=20$ were performed for kinetic energies from $10^{-5}$ to $15000 \mathrm{~cm}^{-1}$. State-to-state rate coefficients for temperatures between 0.1 and $3000 \mathrm{~K}$ are also presented. The comparison of the present results with previous work for lowly-excited rotational levels reveals significant differences. In estimating $\mathrm{HF}-\mathrm{H}_{2}$ rate coefficients, the reduced-potential method is found to be more reliable than the standard reduced-mass approach.

Conclusions. The current state-to-state rate coefficient calculations are the most comprehensive to date for HF-He collisions. We attribute the differences between previously reported data and our results to differences in the adopted interaction potential energy surfaces. The new He rate coefficients can be used in a variety of applications. The estimated $\mathrm{H}_{2}$ and $\mathrm{H}$ collision rates can also augment the smaller datasets previously developed for $\mathrm{H}_{2}$ and electrons.
\end{abstract}

Key words. molecular processes - molecular data - ISM: molecules

\section{Introduction}

Molecular collisions, which are responsible for most of the excitation and reaction processes involving molecules, are important in the interstellar medium (ISM). Collisional excitation and deexcitation processes compete with radiative transitions in populating molecular levels. In cold environments, the important collision partners are $\mathrm{H}_{2}$ and $\mathrm{He}$ because of their large abundances, except in photodissociation regions (PDRs) and diffuse gas where collisions with electrons and $\mathrm{H}$ can become important. Precise laboratory data including collisional deexcitation rate coefficients are required for a range of temperatures to interpret the complicated interstellar spectra of molecular gas not in local thermodynamic equilibrium (LTE). Because of the complexity and difficulty of direct measurements, only limited stateto-state collisional rate coefficients have been measured for systems of astrophysical interest (see, for example, Brunet et al. 2002). Therefore, astrophysical modeling heavily depends on theoretical prediction (e.g., Flower 2007; Faure \& Lique 2012; Wiesenfeld \& Faure 2013; Roueff \& Lique 2013; Yang et al. 2013; Dubernet et al. 2013).

In this paper, we consider hydrogen fluoride (HF), an interstellar molecule containing a halogen element, which was first detected in the ISM by Neufeld et al. (1997). The reactivity

\footnotetext{
* Rate coefficient tables are only available at the CDS via anonymous ftp to cdsarc.u-strasbg. fr $(130.79 .128 .5)$ or via http://cdsarc.u-strasbg.fr/viz-bin/qcat?J/A+A/578/A65
}

of HF is weak, but it may be formed by the exoergic process $\mathrm{F}+\mathrm{H}_{2} \rightarrow \mathrm{HF}+\mathrm{H}$. Experimental rate coefficients for this reaction were recently reported by Tizniti et al. (2014) at temperatures between 10 and $100 \mathrm{~K}$. As a result of its structural stability and radiative properties, the HF molecule can be an alternative tracer to $\mathrm{H}_{2}$ in diffuse regions; $\mathrm{HF}$ is also the main reservoir of fluorine in the ISM (Monje et al. 2011; van der Tak 2012a). Monje et al. (2014) reported observations of HF in two luminous galaxies, NGC 253 and NGC 4945, using the Heterodyne Instrument for the Far-Infrared (HIFI) on the Herschel Space Observatory. With Herschel/HIFI, Neufeld et al. (2010) detected $\mathrm{HF}$ in absorption from the ground rovibrational state. Phillips et al. (2010) presented a detection of the fundamental rotational transition of hydrogen fluoride in absorption toward Orion KL using Herschel/HIFI. The emission in the $j=1 \rightarrow 0$ rotational transition of HF has been observed in the carbon star envelope IRC +10216 by Agúndez et al. (2011). Monje et al. (2011) reported the first detection of HF toward a high-redshift quasar at $z=2.56$, while van der Tak et al. (2012b) presented observations of the HF $j=1 \rightarrow 0$ line in emission towards the Orion Bar.

The HF-He scattering system has been studied theoretically and experimentally (Lovejoy \& Nesbitt 1990; Moszynski et al. 1994, 1996; Chapman et al. 1997; Stoecklin et al. 2003; Reese et al. 2005; Fajin et al. 2006). The availability of ab initio HF-He potential energy surfaces (PESs) has stimulated theoretical studies of HF excitation due to He impact. Lovejoy \& Nesbitt (1990) reported the first experimental study of the near-infrared 
vibrational-rotational spectra of the HeHF and HeDF complexes in a supersonic expansion. The HeHF (HeDF) spectra showed good agreement with the prediction obtained using the HartreeFock dispersion (HFD) type rigid-rotor potential of Rodwell et al. (1981). The spectroscopic data were analyzed and used to probe the isotropic and anisotropic intermolecular potentials of the complexes. The two-dimensional (2D) interaction potential of Moszynski et al. (1994) was developed from ab initio calculations using symmetry-adapted perturbation theory (SAPT). This SAPT potential is in good agreement with the empirical PES of Lovejoy \& Nesbitt (1990). All transition frequencies obtained from the bound-state calculations using the SAPT potential showed excellent agreement with the experimental spectra. The SAPT potential has a global minimum for the linear He-HF arrangement and a secondary minimum for the linear $\mathrm{He}-\mathrm{FH}$ geometry. The accuracy of the SAPT potential was also confirmed by agreement between calculated differential and integral cross sections on a slightly modified SAPT potential and experimental results (Moszynski et al. 1996). Another 2D HF-He potential was reported by Fajin et al. (2006) using the coupled-cluster method with single and double excitations with perturbative triple excitation (CCSD(T)). More recently, a three-dimensional (3D) PES was presented by Stoecklin et al. (2003). This PES was computed using the Brueckner coupled-cluster method with perturbative triples excitations $(\mathrm{BCCD}(\mathrm{T}))$ in the supermolecular approach, and was fitted analytically using a kernel Hilbert space interpolation method. This 3D potential was also used in closecoupling (CC) calculations of pure rotational excitation of $\mathrm{HF}$ in collisions with He by Reese et al. (2005). Cross sections for transitions for rotational levels up to $j=9$ of $\mathrm{HF}$ were calculated for collision energies up to $2000 \mathrm{~cm}^{-1}$. Rate coefficients were obtained from 0.1 to $300 \mathrm{~K}$. However, the PES of Stoecklin et al. predicts global and local minima with well depths of of 43.70 and $25.88 \mathrm{~cm}^{-1}$, respectively, compared to 39.20 and $35.12 \mathrm{~cm}^{-1}$ for the experimentally derived PES of Lovejoy \& Nesbitt (1990).

In this work, explicit quantum-mechanical close-coupling scattering calculations of rotational quenching of HF in collisions with $\mathrm{He}$ at higher levels of rotational excitation were carried out using the SAPT potential of Moszynski et al. (1994). The state-to-state rate coefficients are presented for a wide range of temperatures $(0.1-3000 \mathrm{~K})$, which will aid in modeling rotational spectra of $\mathrm{HF}$ in various astrophysical and atmospheric environments. The computational method is discussed in Sect. 2, and we compare the PESs of Moszynski et al. (1994) and Stoecklin et al. (2003) in Sect. 3. The results are presented in Sect. 4, while astrophysical applications and scaling approaches for estimating $\mathrm{HF}$ deexcitation by $\mathrm{H}_{2}$ and $\mathrm{H}$ collisions are described in Sect. 5.

\section{Quantum-scattering calculations}

We adopted the time-independent quantum-mechanical closecoupling (CC) theory developed by Arthurs \& Dalgarno (1963) for the scattering of a linear rigid-rotor by an $S$-state atom. The state-to-state integral cross section for a transition from an initial rotational state $j$ to a final rotational state $j^{\prime}$ can be expressed as

$$
\begin{aligned}
\sigma_{j \rightarrow j^{\prime}}\left(E_{j}\right)= & \frac{\pi}{(2 j+1) k_{j}^{2}} \sum_{J=0}(2 J+1) \\
& \times \sum_{l=|J-j| l^{\prime}=\left|J-j^{\prime}\right|}^{J+j} \sum_{j+j^{\prime}}^{J+j^{\prime}} \delta_{l l^{\prime}}-\left.S_{j j^{\prime} l l^{\prime}}^{J}\left(E_{j}\right)\right|^{2},
\end{aligned}
$$

Table 1. Computed rotational excitation energies $\left(\mathrm{cm}^{-1}\right)$ of HF.

\begin{tabular}{cccc}
\hline \hline$j$ & $E_{j}$ & $j$ & $E_{j}$ \\
\hline 0 & 0.00000 & 16 & 5540.34896 \\
1 & 41.89886 & 17 & 6210.52398 \\
2 & 125.64498 & 18 & 6914.70306 \\
3 & 251.13516 & 19 & 7651.95740 \\
4 & 418.21460 & 20 & 8421.30660 \\
5 & 626.67690 & 21 & 9221.71866 \\
6 & 876.26406 & 22 & 10052.10998 \\
7 & 1166.66648 & 23 & 10911.34536 \\
8 & 1497.52296 & 24 & 11798.23800 \\
9 & 1868.42070 & 25 & 12711.54950 \\
10 & 2278.89530 & 26 & 13649.98986 \\
11 & 2728.43076 & 27 & 14612.21748 \\
12 & 3216.45948 & 28 & 15596.83916 \\
13 & 3742.36226 & 29 & 16602.41010 \\
14 & 4305.46830 & 30 & 17627.43390 \\
15 & 4905.05520 & & \\
\hline
\end{tabular}

Notes. $E_{j}=B_{\mathrm{e}} j(j+1)-D j^{2}(j+1)^{2}$, where $B_{\mathrm{e}}$ and $D$ are the rotational constant and the centrifugal distortion constant of $\mathrm{HF}$, respectively.

where $\mathbf{j}$ and $\mathbf{l}$ are the rotational angular momentum of the HF molecule and the orbital angular momentum of the collision complex, respectively. The total angular momentum $\mathbf{J}$ is given by $\mathbf{J}=\mathbf{I}+\mathbf{j} . S_{j j^{\prime} l l^{\prime}}^{J}$ is an element of the scattering matrix, which is obtained by solving coupled-channel equations and employing the usual boundary conditions. $k_{j}=\sqrt{2 \mu E_{j}} / \hbar$ denotes the wave vector for the initial channel, $E_{j}$ is the kinetic energy for the initial channel, and $\mu$ the reduced mass of the HF-He system. The total quenching cross section from an initial state $j$ can be obtained by summing the state-to-state cross sections $\sigma_{j \rightarrow j^{\prime}}\left(E_{j}\right)$ over all final $j^{\prime}$ states, where $j^{\prime}<j$.

The quantum-scattering code MOLSCAT (Hutson \& Green 1994) was applied in the close-coupling calculations. The propagation in $R$ was carried out to $50 \AA$ with the coupled-channel equations solved using the modified log-derivative Airy propagator (Alexander \& Manolopoulos 1987). To ensure convergence in the cross-section calculations, at least five to ten closed channels in the basis and a sufficient number of partial waves were included. HF rotational energy levels are given in Table 1, which were obtained using the rotational constant $B_{\mathrm{e}}=20.953 \mathrm{~cm}^{-1}$ (Irikura 2007) and the centrifugal distortion constant $D=0.0021199 \mathrm{~cm}^{-1}$ (Coxon \& Hajigeorgiou 1990). The $\mathrm{CC}$ calculations were performed for collision energies ranging from $10^{-5}$ to $15000 \mathrm{~cm}^{-1}$ with $\mu=3.3353 \mathrm{u}$.

The rate coefficients for rotational transitions can be computed numerically by thermally averaging the corresponding cross sections over a Maxwellian kinetic energy distribution

$k_{j \rightarrow j^{\prime}}(T)=\left(\frac{8}{\pi \mu \beta}\right)^{1 / 2} \beta^{2} \int_{0}^{\infty} E_{j} \sigma_{j \rightarrow j^{\prime}}\left(E_{j}\right) \exp \left(-\beta E_{j}\right) \mathrm{d} E_{j}$,

where $T$ is the temperature, $\beta=\left(k_{\mathrm{B}} T\right)^{-1}$, and $k_{\mathrm{B}}$ is Boltzmann's constant. 

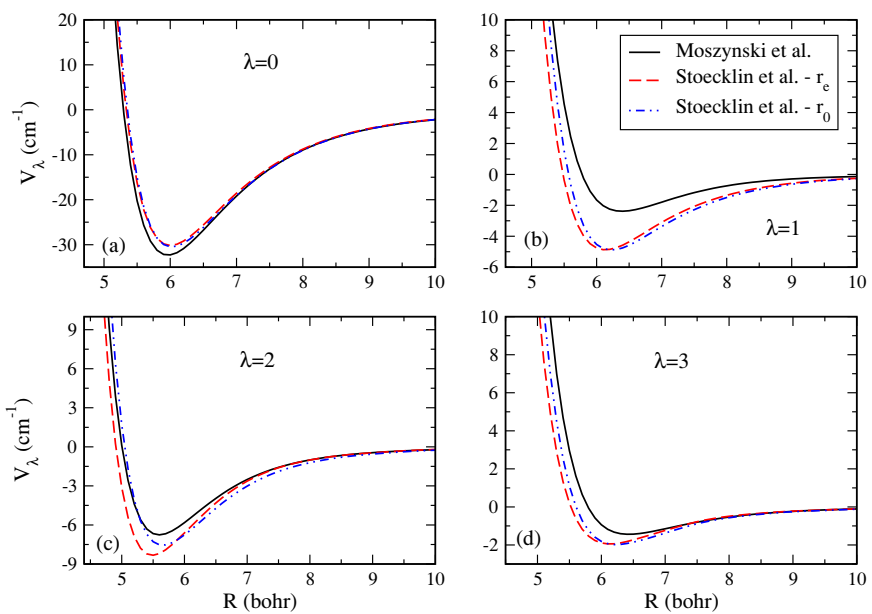

Fig. 1. Potential expansion terms $v_{\lambda}, \lambda=0,1,2$, and 3, for the HF-He PESs of Moszynski et al. (1994) and Stoecklin et al. $r_{\mathrm{e}}$ and $r_{0}$ are used to obtain 2D PESs for Stoecklin et al. (2003), while the Moszynski et al. PES was constructed for $r_{\mathrm{e}}$.

\section{Comparison of potential energy surfaces}

In the rigid-rotor scattering calculations, the interaction potential of HF-He, $V(R, \theta)$, was expanded in terms of Legendre polynomials,

$V(R, \theta)=\sum_{\lambda=0}^{\lambda_{\max }} v_{\lambda}(R) P_{\lambda}(\cos \theta)$,

where $P_{\lambda}$ are Legendre polynomials of order $\lambda, R$ is the distance between the HF center of mass and the He atom, and $\theta$ the angle between $\mathbf{R}$ and the HF molecular axis. The angular dependence of the interaction potential was expanded to $\lambda_{\max }=20$.

In Fig. 1, the first four components of $v_{\lambda}(R), \lambda=0,1,2$, and 3 are plotted as a function of $R$ for the PESs of Moszynski et al. (1994) and Stoecklin et al. (2003). To obtain a 2D rigidrotor PES, the optimal approach is to average the 3D PES over the ground-state vibrational function of the diatomic molecule (Faure et al. 2005; Kalugina et al. 2014). However, in this work, we mainly used the 2D PES of Moszynski et al. (1994), which was obtained at $r_{\mathrm{e}}$, to calculate the rotational quenching cross sections and rate coefficients. For the 3D PES of Stoecklin et al., two values of the HF bond length, the equilibrium distance $r_{\mathrm{e}}=1.7328 a_{0}$ and the vibrationally averaged bond distance for the ground vibrational state $r_{0}=1.767 a_{0}$ (Zhang \& Zhang 1993), were used to compute $v_{\lambda}(R)$. Comparing the plots, we can see that differences between the PESs of Moszynski et al. and Stoecklin et al. are apparent, particularly for components $\lambda=1$ and 2. In the case of the PES of Stoecklin et al., except for $\lambda=0$, some differences can be seen between the curves for $R$ less than $6 a_{0}$ owing to the different values of the HF bond length. Therefore, one can expect discrepancies to arise in scattering calculations performed on the two different PESs. We recall that the Moszynski et al. PES agrees very well with the experimentally deduced surface of Lovejoy \& Nesbitt (1990).

\section{Results and discussion}

\subsection{State-to-state and total quenching cross sections}

We performed calculations of the state-to-state quenching cross sections for initial HF rotational states of $j=1,2, \ldots, 20$ using

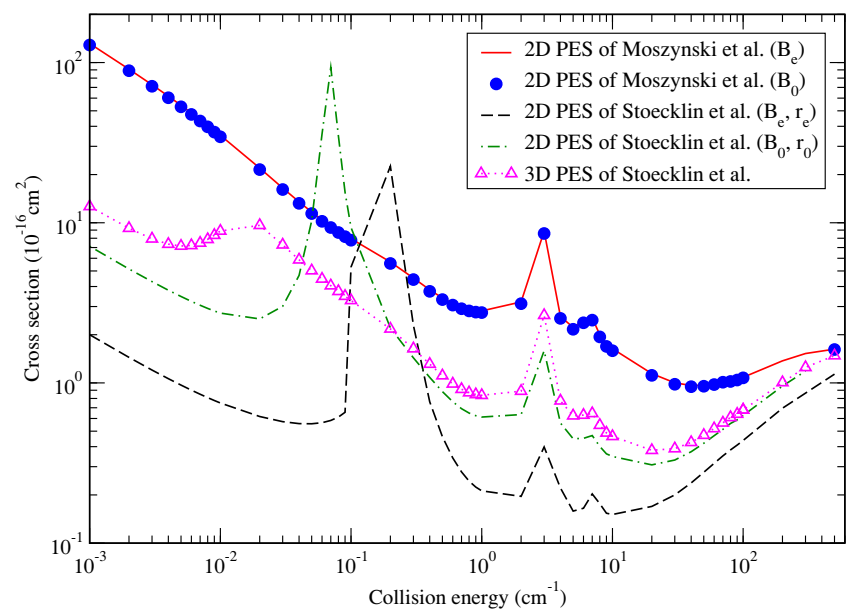

Fig. 2. State-to-state rotational quenching cross sections from initial state $j=1$ of HF in collisions with He.

the PES of Moszynski et al. (1994) ${ }^{1}$. To evaluate the accuracy of the current computed cross section and to compare with the results obtained using the PES of Stoecklin et al. (2003), calculations of state-to-state quenching cross sections from $j=1$ and 3 were also performed using the PES of Stoecklin et al. ${ }^{2}$. In the rigid-rotor approximation calculations carried out on the PES of Stoecklin et al., the 2D PESs are obtained by fixing the HF bond distance at $r_{\mathrm{e}}$ and $r_{0}$. Correspondingly, rotational constants of HF $B_{\mathrm{e}}=20.9537 \mathrm{~cm}^{-1}$ (Irikura 2007) and $B_{0}=B_{\mathrm{e}}-1 / 2 \alpha_{\mathrm{e}}=$ $20.5570 \mathrm{~cm}^{-1}$ were used in the cross-section calculations, where the vibration-rotation interaction constant $\alpha_{\mathrm{e}}=0.7934 \mathrm{~cm}^{-1}$ (Irikura 2007). As examples, the state-to-state quenching cross sections from initial states $j=1$ and 3 are presented in Figs. 2 and 3 , respectively. In the case of quenching $j=1 \rightarrow 0$, Fig. 2 illustrates that there are significant differences between the cross sections obtained using the PESs of Moszynski et al. and Stoecklin et al. that are due to the different structures of the two PESs, as shown in Fig. 1. For the PES of Stoecklin et al., the cross sections calculated using $\left(B_{0}, r_{0}\right)$ agree better with the results obtained using their 3D potential. A number of resonances in the cross section, which occur for low collision energies associated with the van der Waals wells, demonstrate their sensitivity to the adopted PES.

The PES of Moszynski et al., used in our calculations, was constructed by fixing the HF bond distance at its equilibrium value $r_{\mathrm{e}}$. We used the rotational constant $B_{\mathrm{e}}$ to evaluate the HF rotational energy levels (see Table 1). However, to study the effect of the rotational constant on the cross sections, we carried out cross-section calculations from initial states $j=1,3$, and 10 using the rotational constant $B_{0}$. Figures 2 and 3 show that for the PES of Moszynski et al., the state-to-state quenching cross sections from initial $j=1$ and 3 using $B_{\mathrm{e}}$ and $B_{0}$ are nearly

1 All state-to-state deexcitation cross sections and rate coefficients for HF-He are available on the UGA Excitation Database website (www.physast.uga.edu/amdbs/excitation). The rate coefficients are also available in the BASECOL (Dubernet et al. 2013) and the Leiden Atomic and Molecular Database (LAMDA; Schöier et al. 2005) formats. In addition, estimates for $\mathrm{HF}-\mathrm{H}_{2}$ and $\mathrm{HF}-\mathrm{H}$ rate coefficients obtained by the reduced-potential scaling approach, described below, are included in the new LAMDA file along with the original data from the LAMDA website.

2 The rotational deexcitation cross sections displayed in Reese et al. (2005) were found to be discrepant and not consistent with rate coefficients given in the same paper as confirmed by Stoecklin (priv. comm.). 


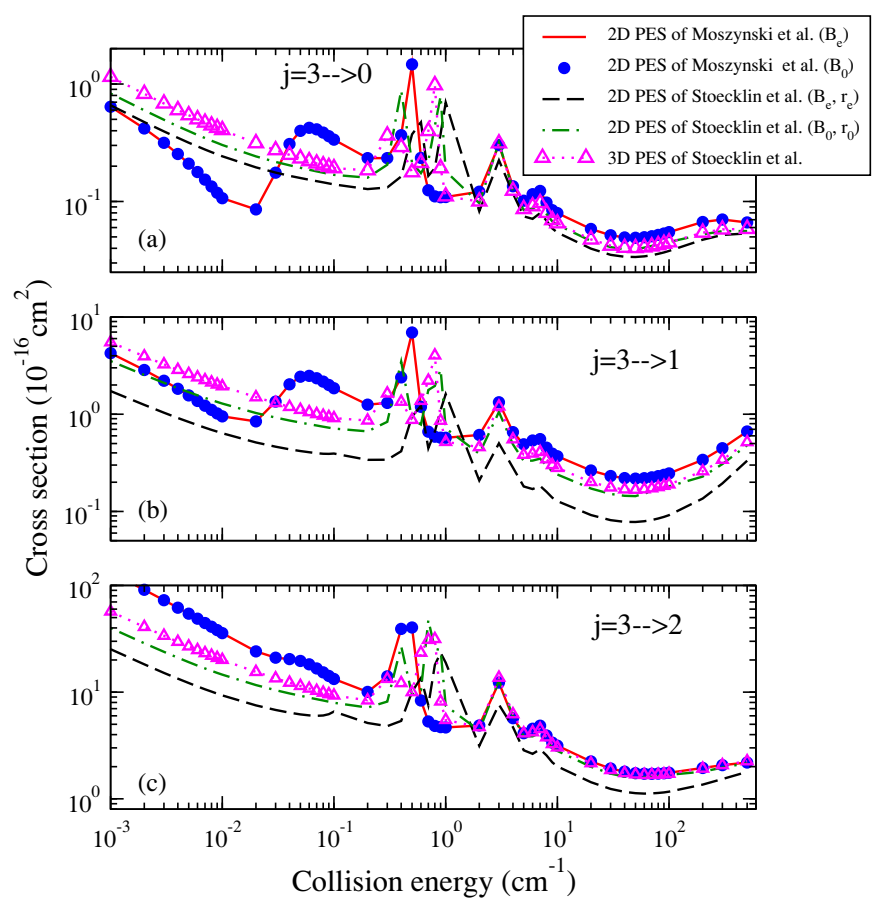

Fig. 3. State-to-state rotational quenching cross sections from initial state $j=3$ of HF in collisions with He.

identical. For state-to-state quenching cross sections from initial $j=10$ (not shown), the differences between the results obtained using $B_{\mathrm{e}}$ and $B_{0}$ are generally lower than $10 \%$.

In Fig. 3 we compare the state-to-state quenching cross section from initial state $j=3$. As observed in the case of $j=1$, the cross sections display resonances in the intermediate energy region from $\sim 0.01 \mathrm{~cm}^{-1}$ to $\sim 10 \mathrm{~cm}^{-1}$ due to quasibound levels supported by the attractive part of the interaction potential. Importantly, for astrophysical applications, the properties of the resonances influence the quenching rate coefficients at low temperatures. In contrast to initial $j=1$, the difference between the cross sections obtained on the two PESs is smaller. In particular, for the PES of Stoecklin et al. (2003) the results using $\left(B_{0}, r_{0}\right)$ are similar to the results using the $3 \mathrm{D}$ potential for collision energies higher than $1.0 \mathrm{~cm}^{-1}$. As can be seen, the deexcitation process from $j=3$ is dominated by the $\Delta j=-1$ transition. Furthermore, the computed cross sections show that the $\Delta j=j^{\prime}-j=-1$ transition dominates the deexcitation for all $j$, and the cross sections generally increase with increasing $j^{\prime}$, with that for $j^{\prime}=0$ being the smallest.

The total quenching cross section from a given initial level $j$ can be computed by summing over all final states $j^{\prime}$. In Fig. 4 the total quenching cross sections from selected initial levels $j=2,4,6, \ldots, 18$, and 20 are displayed. Generally, the total quenching cross sections have similar behavior, decreasing with $j$ for $E_{j} \leq 50 \mathrm{~cm}^{-1}$, but differences result for small $j$ at high energy that are due to a limited number of final exit channels. Each of the cross sections exhibit the behavior predicted by the Wigner (1948) threshold law at ultra-low collision energies below $\sim 10^{-3} \mathrm{~cm}^{-1}$, where only $s$-wave scattering contributes and the cross sections vary inversely with the relative velocity. In the intermediate energy region, between 0.1 and $10 \mathrm{~cm}^{-1}$, the cross sections display scattering resonances, but they reveal somewhat different structures depending on the initial rotational state $j$ between 0.05 and $1 \mathrm{~cm}^{-1}$. Except for $j=2$, the total deexcitation cross sections decrease to a global minimum near $50 \mathrm{~cm}^{-1}$.

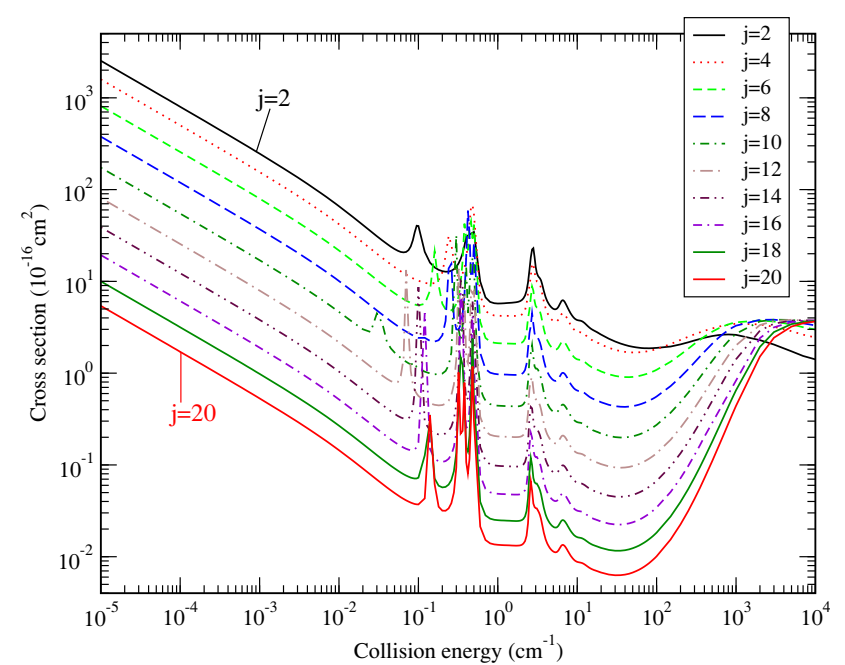

Fig. 4. Total deexcitation cross sections from initial states $j=2,4,6,8$, $10,12,14,16,18$, and 20 of HF in collisions with He with the PES of Moszynski et al. (1994).

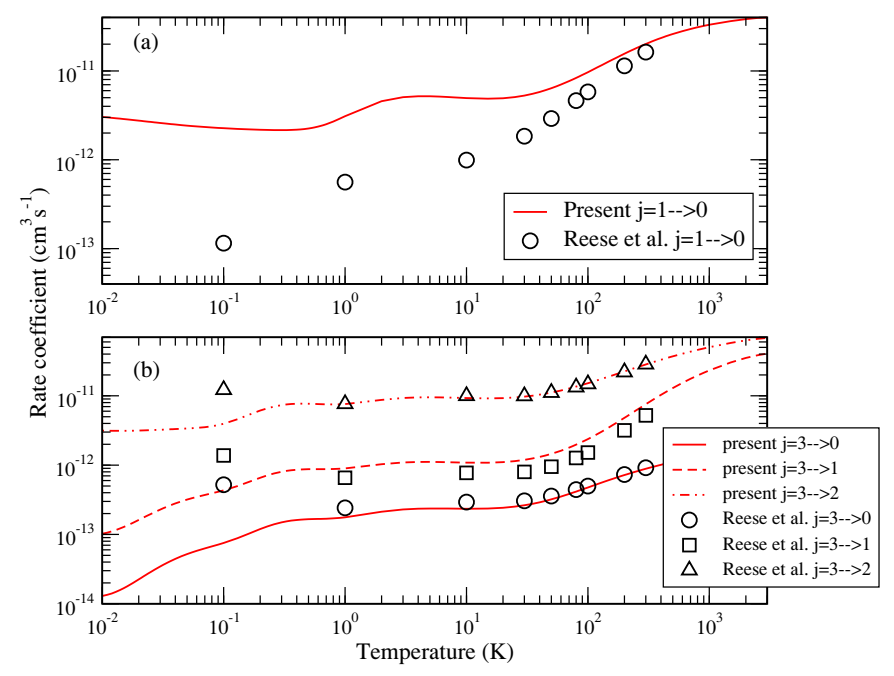

Fig. 5. State-to-state rotational quenching rate coefficients from initial states $j=1$ and 3 of HF that are due to collisions with He. a) $j=1$; b) $j=3$. Lines: present results with the PES of Moszynski et al. (1994); symbols: Reese et al. (2005) with the PES of Stoecklin et al. (2003).

\subsection{State-to-state quenching rate coefficients}

The quenching rate coefficients can be computed by averaging the appropriate cross sections over a Maxwell-Boltzmann distribution of collision energy $E_{j}$, as given by Eq. (2). The state-tostate quenching rate coefficients for initial $\mathrm{HF}$ rotational states of $j=1,2, \ldots, 20$ were calculated using $B_{\mathrm{e}}$ and the PES of Moszynski et al. (1994). However, to our knowledge, there are no published experimental rate coefficients available. Our rate coefficients, computed using the PES of Moszynski et al., are only compared with the theoretical results of Reese et al. (2005), which were obtained over the limited temperature range of 0.1 to $300 \mathrm{~K}$. As examples, Figs. 5-7 present selected stateto-state quenching rate coefficients from initial rotational levels $j=1,3,5$, and 9. Except for deexcitation from $j=1$, the current results generally follow similar trends with that of Reese et al., which were computed using the 3D potential of Stoecklin et al. (2003). However, at $0.1 \mathrm{~K}$, the rate coefficients of Reese et al. are always significantly larger than the current results for all transitions, except for $j=1$. 
B. Yang et al.: Collisional quenching of highly rotationally excited HF

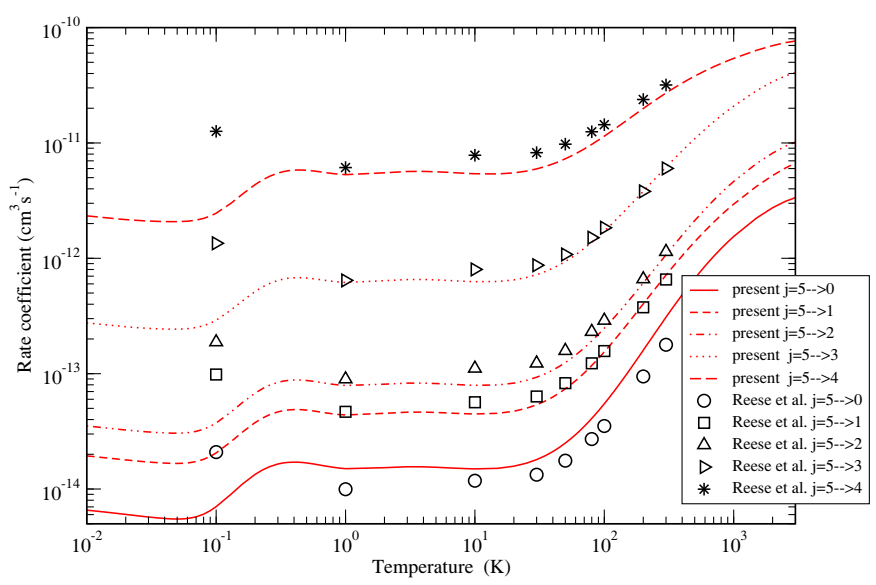

Fig. 6. State-to-state rotational quenching rate coefficients from initial states $j=5$ of HF that are due to collisions with He. Lines: present results with the PES of Moszynski et al. (1994); symbols: Reese et al. (2005) with the PES of Stoecklin et al. (2003).

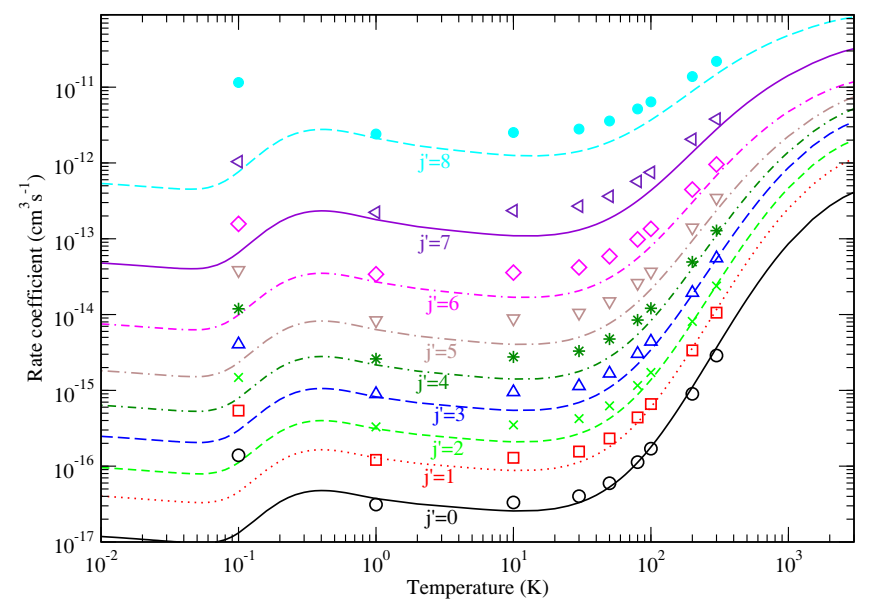

Fig. 7. Same as Fig. 6, but for initial rotational state $j=9$.

For initial state $j=1$, Fig. 5a shows that the current rate coefficients are larger than the results of Reese et al. As for initial state $j=3$ shown in Fig. $5 \mathrm{~b}$, for temperatures above $1 \mathrm{~K}$, the current state-to-state rate coefficients agree reasonably well with that of Reese et al., although our results are somewhat larger for the $j=3 \rightarrow 1$ transition. State-to-state quenching rate coefficients from initial states $j=5$ and 9 are compared in Figs. 6 and 7, respectively, with the results of Reese et al. Except for the deexcitation to the final state $j^{\prime}=0$ and for $0.1 \mathrm{~K}$, the current rate coefficients are smaller than those of Reese et al., similar to what is found for $j=3$. For each dominant deexcitation transition, $\Delta j=j^{\prime}-j=-1, j=1,2, \ldots, 9$, at a temperature of $50 \mathrm{~K}$, we compare the percent differences between our rate coefficients and the results of Reese et al. (2005). In Fig. 8, the percent differences are displayed as a function of initial rotational state $j$. The percent difference is near zero for $j=3$, but the differences vary from $20 \%$ to $75 \%$ for all other $j$ values.

For illustration, in Fig. 9 we present the state-to-state deexcitation rate coefficients for temperatures ranging from $0.1 \mathrm{~K}$ to $3000 \mathrm{~K}$ for initial states $j=10$ and 20 . Over the whole temperature range considered, the rate coefficients generally increase with increasing temperature for all transitions. Furthermore, the rate coefficients clearly decrease with increasing $|\Delta j|=\left|j^{\prime}-j\right|$ with the $\Delta j=-1$ transitions dominant.

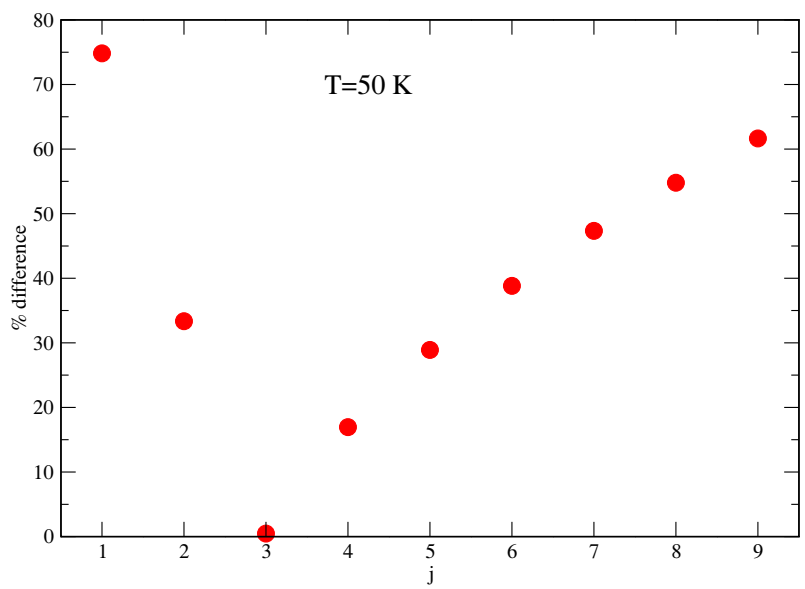

Fig. 8. Percent difference of HF-He rate coefficients for the deexcitation of level $j$ for the dominant transition $\Delta j=-1$ at $50 \mathrm{~K}$ between current results using the PES of Moszynski et al. (1994) and the results of Reese et al. (2005) with the PES of Stoecklin et al. (2003).

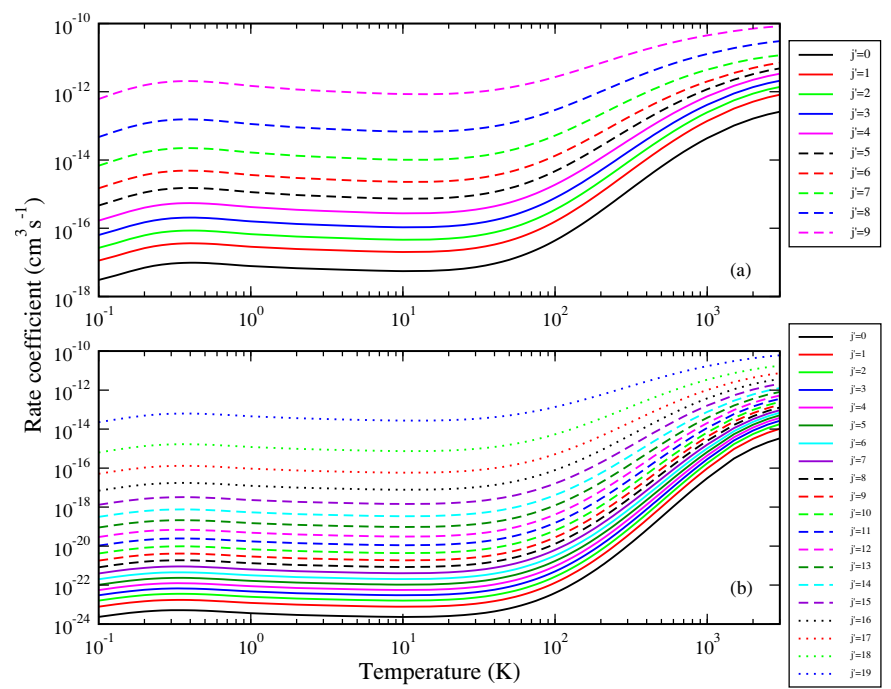

Fig. 9. State-to-state rotational deexcitation rate coefficients from initial state $j=10$ and $j=20$ of HF in collisions with He using the PES of Moszynski et al. (1994). a) $j=10$; b) $j=20$.

\section{Applications}

As highlighted in the Introduction, HF has been observed in both emission and absorption in a variety of astronomical environments that may be characterized by diverse physical conditions. As a consequence, the rotational levels of HF may be populated by different, or multiple, mechanisms leading to spectra differing from LTE. For the UV-irradiated environment of the Orion Bar, van der Tak et al. (2012b) considered electrons and $\mathrm{H}_{2}$ as possible impactors for inelastic collisional excitation. However, given that the Orion Bar is a prototypical photodissociation region (PDR), the abundances of both $\mathrm{H}$ and $\mathrm{He}$ are most likely higher than or comparable to that of $\mathrm{H}_{2}$. In many PDR enviroments, collisions due to all four colliders may need to be considered.

For a variety of reasons, it is often the case that excitation rate coefficients for a molecular species may only be available for He collisions, as performed in the current work. In such instances, a common practice is to estimate $\mathrm{H}_{2}$, and occasionally $\mathrm{H}$, collisional excitation rate coefficients using $\mathrm{He}$ data by scaling by the square root of the ratio of the collision systems' 


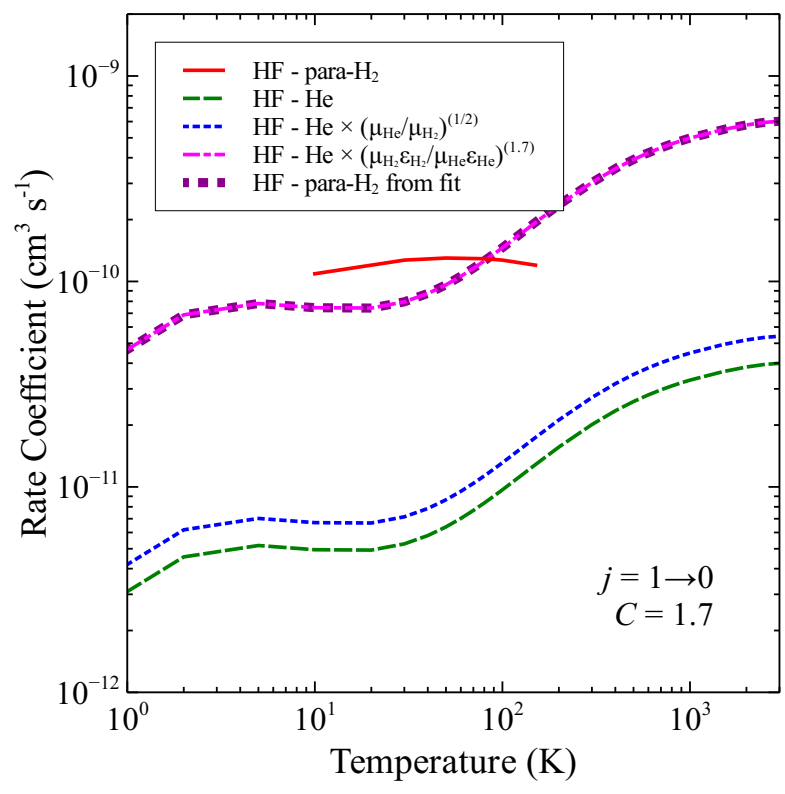

Fig. 10. HF-para- $\mathrm{H}_{2}$ (Guillon \& Stoecklin 2012) and HF-He rate coefficients (current results) compared to $\mathrm{HF}-$ para- $\mathrm{H}_{2}$ rate coefficients deduced by various scaling approaches for the $j=1 \rightarrow 0$ transition. See text for discussion.

reduced masses, here a factor of 1.4 for $\mathrm{H}_{2}$. Walker et al. (2014) demonstrated on both theoretical and numerical grounds that this standard reduced-mass scaling approach is typically invalid. We therfore do not recommend here that such an approach be adopted with the present HF-He rate coefficients. Fortunately, explicit $\mathrm{HF}-\mathrm{H}_{2}$ rate coefficients for some rotational quenching transitions of the HF ground vibrational state have been computed by Guillon \& Stoecklin (2012).

\subsection{Prediction of HF rate coefficients by scaling}

Given the availability of $\mathrm{HF}-\mathrm{H}_{2}$ and $\mathrm{HF}-\mathrm{He}$ collisional data, we can test various scaling methods, including the reduced-potential approach introduced in Walker et al. (2014). In the reducedpotential method, the collisional data are scaled by the reduced potentials $\mu_{X} \varepsilon_{X}$ according to

$k_{j \rightarrow j^{\prime}}^{Z}(T)=\left(\frac{\mu_{Z} \varepsilon_{Z}}{\mu_{Y} \varepsilon_{Y}}\right)^{C} k_{j \rightarrow j^{\prime}}^{Y}(T)$,

where $\mu$ is the reduced mass of the HF- $X$ system, $\varepsilon_{X}$ is the van der Waals minimum of the HF- $X$ system, and $C$ is a phenomenological exponent. $X=Y$ is usually He, with $X=Z$ typically para- $\mathrm{H}_{2}$. The reduced-potential and standard reduced-mass approaches are compared in Fig. 10 for the HF rotational deexcitation transition $j=1 \rightarrow 0$. Standard reduced-mass scaling results in an estimate for para- $\mathrm{H}_{2}$ collisions that is a factor of 10 too small, while the reduced-potential method with an exponent of $C=1.7$ agrees well.

Given its better performance, the reduced-potential scaling approach of Walker et al. (2014) was therefore used to predict unknown rate coefficients for para- $\mathrm{H}_{2}$ and $\mathrm{H}$ colliders with $\mathrm{HF}$. We adopted the He collision data computed here and the smaller set of para- $\mathrm{H}_{2}$ rate coefficients for $j \leq 5$ calculated by Guillon $\&$ Stoecklin (2012) for $T=10-150 \mathrm{~K}$. The rate coefficients are very sensitive to the presence of quasibound resonances over this temperature range, which may partially be accounted for with the reduced-potential approach since it takes into the account
Table 2. Optimized values of $C$ and their respective normalized root-mean-square deviations (NRMSD, in \%) for collisional deexcitation transitions of $\mathrm{HF}$ with $\mathrm{H}_{2}$ scaled via the standard reduced-mass (rm) and reduced-potential (rp) methods from HF-He collisional rate coefficients.

\begin{tabular}{cccc}
\hline \hline$j \rightarrow j^{\prime}$ & $C$ & NRMSD $_{\text {rm }}$ & NRMSD $_{\text {rp }}$ \\
\hline $1-0$ & 1.7 & 1058.85 & 34.90 \\
$2-0$ & 1.3 & 299.75 & 22.66 \\
$2-1$ & 0.6 & 161.28 & 15.42 \\
$3-0$ & -0.2 & 53.29 & 7.55 \\
$3-1$ & -0.1 & 30.66 & 8.77 \\
$3-2$ & -0.2 & 62.78 & 3.77 \\
$4-0$ & -1.6 & 69.89 & 8.14 \\
$4-1$ & -0.3 & 42.20 & 16.48 \\
$4-2$ & -1.3 & 73.59 & 13.37 \\
$4-3$ & -0.8 & 90.81 & 11.69 \\
$5-0$ & -1.2 & 54.55 & 18.68 \\
$5-1$ & -2.0 & 61.64 & 13.50 \\
$5-2$ & -0.5 & 44.98 & 16.22 \\
$5-3$ & -1.3 & 66.96 & 6.48 \\
$5-4$ & -1.2 & 86.17 & 7.98 \\
\hline
\end{tabular}

the different interaction well-depths. Compared to the reducedmass scaling technique, where the rate coefficients simply scale as the square root of the ratio of the reduced masses, the reducedpotential scaling approach offers an improvement for predicted $\mathrm{HF}$ rate coefficients, much like the improved $\mathrm{CO}$ and $\mathrm{H}_{2} \mathrm{O}$ rate coefficient predictions of Walker et al. (2014). While the value of $C$ for $\mathrm{CO}$ rate coefficients ranged from -0.2 to 1.3 and from 0.5 to 1.2 for $\mathrm{H}_{2} \mathrm{O}$, it was noted that symmetries were involved in obtaining the best value of $C$.

The accuracy of the reduced-potential scaling approach can be further tested here with the heteronuclear molecule HF. The normalized root-mean-square deviation (NRMSD), $\sigma_{\text {norm }}$, quantifies the residual variance between the calculated $\mathrm{H}_{2}$ rate coefficients, $k_{\text {calc }}$, and those scaled from $\mathrm{He}, k_{\text {scale }}$, and is given by

$\sigma_{\text {norm }}=\frac{\sqrt{\frac{\sum_{T=i}^{N}\left(k_{\text {scale }}(T)-k_{\text {calc }}(T)\right)^{2}}{N}}}{k_{\max }-k_{\min }}$,

where $N$ is the number of temperature data points and $k_{\max }$ and $k_{\min }$ are the values of the maximum and minimum rate coefficients. The resulting NRMSD percentages for the $\mathrm{H}_{2}$ rate coefficient predictions for both standard reduced-mass scaling and reduced-potential scaling is given in Table 2 and Fig. 11 for the first fifteen transitions of $\mathrm{HF}$ at $50 \mathrm{~K}$. When comparing the NRMSD for both methods for each transition of HF, the reduced-potential scaling predictions exhibit less residual variance in all fifteen transitions with a mean of $14 \%$ in its ability to reproduce the explicit $\mathrm{HF}-\mathrm{H}_{2}$ calculations as computed by Guillon \& Stoecklin (2012). In many cases, the NRMSD for standard reduced-mass scaling exceeds $100 \%$, while the reduced-potential approach gives NRMSD $<35 \%$. A linear leastsquares analysis was then performed, using the first fifteen transitions of $\mathrm{HF}$, for each $j^{\prime}$ and the resulting linear functions are plotted in Fig. 12. Except for $j^{\prime}=2$, the lines converge around $|\Delta j|=6$ and $C=-3$. For $|\Delta j|=1$, the values of $C$ decrease linearly with $j^{\prime}$ and are listed in Table 3 for $j^{\prime}=0-4$. The optimal values for $C$, valid for all temperatures, were forced to exactly reproduce the reduced-potential scaling result for the dominant $\Delta j=-1$ transitions.

To use the reduced-potential approach in astrophysical applications, however, one needs estimates of $C$ when data for 
B. Yang et al.: Collisional quenching of highly rotationally excited HF

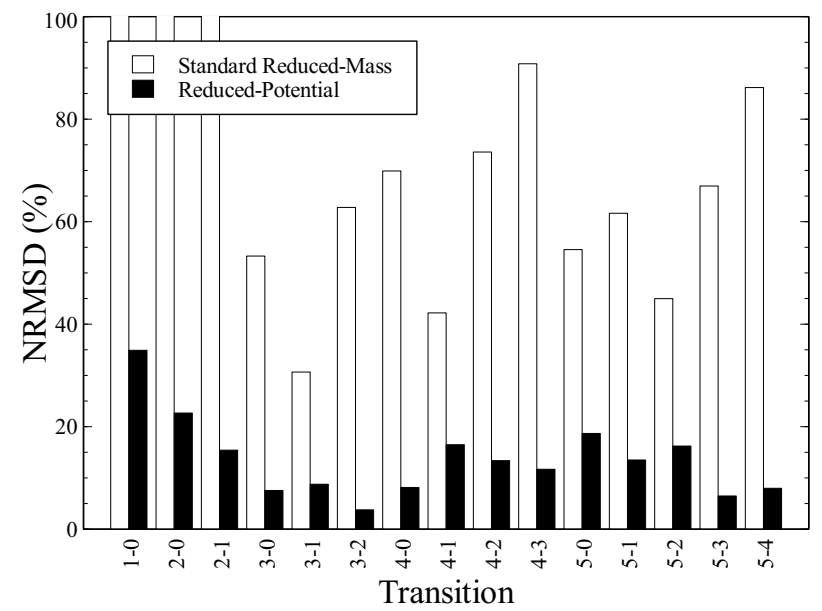

Fig. 11. Normalized root-mean-square deviation (NRMSD) in standard reduced-mass scaling and reduced-potential scaling for 15 transitions of HF-para- $\mathrm{H}_{2}$, truncated at $100 \%$.

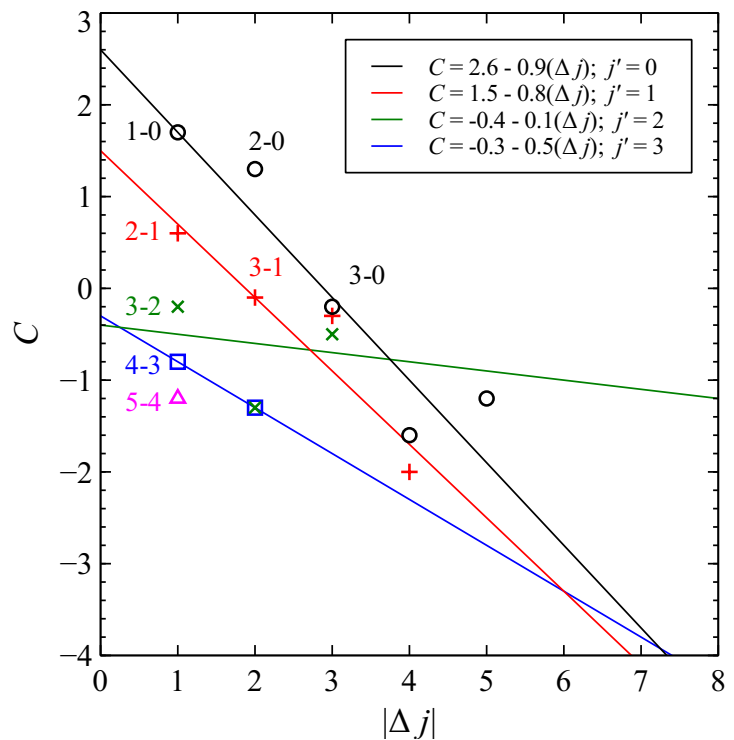

Fig. 12. Phenomological constant $C$ as a function of $|\Delta j|$. A linear leastsquares analysis was performed for each $j^{\prime}$ and the resulting linear functions are plotted. Note the convergence of these functions (except for $j^{\prime}=2$ ) around $|\Delta j|=6$ and $C=-3$. Symbols correspond to $C$ determined for actual HF-para- $\mathrm{H}_{2}$ and HF-He data.

the impactor of interest $Z$, e.g. para- $\mathrm{H}_{2}$, are unknown. Using the information from Fig. 12, the slope and $y$-intercept can be obtained for each transition and the value of $C$ predicted as given in Fig. 13 and Table 3 for $j^{\prime}=5-20$. The best prediction for transitions with $|\Delta j| \geq 6$ is obtained with $C=-3$. Rate coefficients for the transitions of $\mathrm{H}_{2}$ with $j<5$ are reproduced reasonably well by the reduced-potential approach with $C$ decreasing with increasing $|\Delta j|$ and increasing $j^{\prime}$. Figures 10 and 14 compare rate coefficient estimates using the fit prediction of $C$ to direct reduced-potential and reduced-mass values for select transitions. Weak transitions are scaled with $C=-3$, and since these rate coefficients are several orders of magnitude smaller than the dominant transitions, larger error in the predicted values is acceptable.

Given that the available $\mathrm{HF}-\mathrm{H}_{2}$ rate coefficients are limited to $j \leq 5$ (Guillon \& Stoecklin 2012), we used the reducedpotential scaling method predictions for $C$ and the current HFHe data to estimate HF rotational deexcitation for $j=6-20$
Table 3. HF reduced-potential fitting parameters.

\begin{tabular}{cccc}
\hline \hline$j^{\prime}$ & $C$ & $\Delta C$ & Slope \\
\hline 0 & 1.7 & 1.1 & -0.94 \\
1 & 0.6 & 0.8 & -0.72 \\
2 & -0.2 & 0.6 & -0.56 \\
3 & -0.8 & 0.4 & -0.44 \\
4 & -1.2 & 0.2 & -0.36 \\
\hline 5 & -1.4 & 0 & -0.32 \\
6 & -1.5 & 0 & -0.30 \\
7 & -1.6 & 0 & -0.28 \\
8 & -1.7 & 0 & -0.26 \\
9 & -1.8 & 0 & -0.24 \\
10 & -1.9 & 0 & -0.22 \\
11 & -2 & 0 & -0.20 \\
12 & -2.1 & 0 & -0.18 \\
13 & -2.2 & 0 & -0.16 \\
14 & -2.3 & 0 & -0.14 \\
15 & -2.4 & 0 & -0.12 \\
16 & -2.5 & 0 & -0.10 \\
17 & -2.6 & 0 & -0.08 \\
18 & -2.7 & 0 & -0.06 \\
19 & -2.8 & 0 & -0.04 \\
20 & -2.9 & 0 & -0.02
\end{tabular}

Notes. The lower states $j^{\prime}$, the optimized values of $C$, the change in $C(\Delta C)$, and the slope of the derived linear functions.

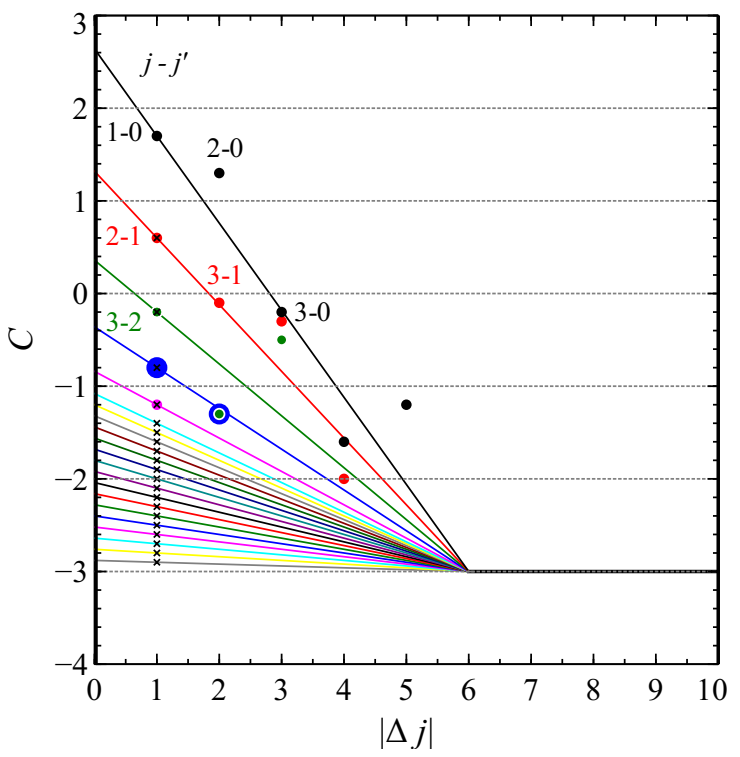

Fig. 13. Phenomological constant $C$ as a function of $|\Delta j|$. As a result of the linear decrease of $C$ with $j^{\prime}$ and the convergence around $|\Delta j|=6$ and $C=-3$, the slope and $y$-intercept can be obtained for each transition and the value of $C$ can be predicted. Symbols and fit lines are for $j^{\prime}=$ $0-4$, same as in Fig. 12.

for para- $\mathrm{H}_{2}$ collisions. Furthermore, as there is a complete lack of $\mathrm{HF}$ rotational excitation data for $\mathrm{H}$ impact, we extended the reduced-potential scaling approach to estimate HF-H deexcitation rate coefficients from the current $\mathrm{HF}-\mathrm{He}$ rate coefficients with the trend in $C$ taken from Fig. 13. In using the reducedpotential approach, the following parameters were adopted for $\mathrm{HF}$ collisions with $\mathrm{He}, \mathrm{H}_{2}$, and $\mathrm{H}: \mu_{\mathrm{He}}=3.3353 \mathrm{u}, \mu_{\mathrm{H}_{2}}=1.818$ $\mathrm{u}, \mu_{\mathrm{H}}=0.9596 \mathrm{u}, \varepsilon_{\mathrm{He}}=39.68 \mathrm{~cm}^{-1}$ (Moszynski et al. 1994), $\varepsilon_{\mathrm{H}_{2}}=359.0 \mathrm{~cm}^{-1}$ (Guillon et al. 2008), and $\varepsilon_{\mathrm{H}}=100.0 \mathrm{~cm}^{-1}$ (Stark \& Werner 1996). All rate coefficient data are available in the LAMDA format, as mentioned in footnote 1 . The availability 


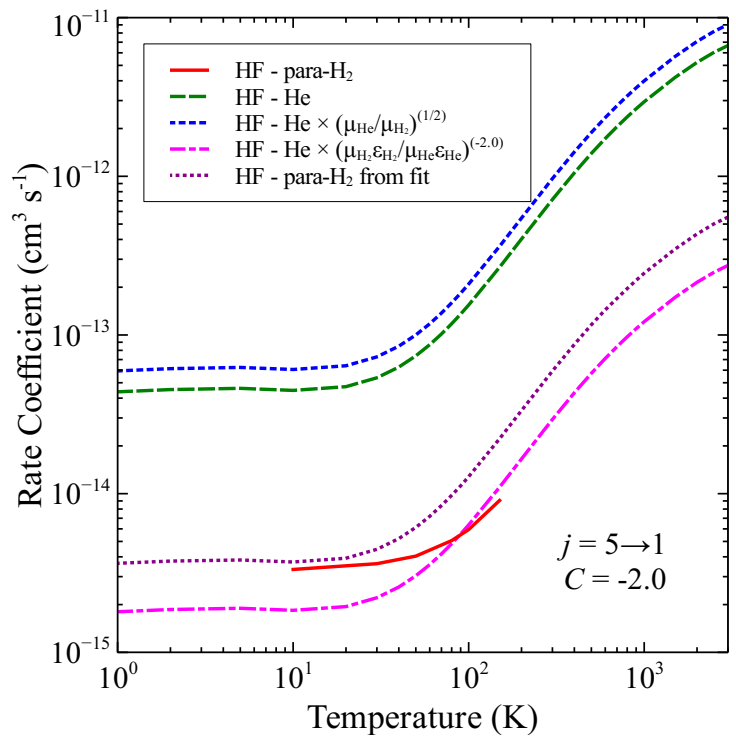

Fig. 14. Same as Fig. 10, but for the $j=5 \rightarrow 1$ transition.

of a complete set of HF rotational quenching rate coefficients due to collisions with $\mathrm{H}_{2}, \mathrm{He}, \mathrm{H}$, and $\mathrm{e}^{-}$will allow for detailed modeling of HF rotational emission lines in a variety of environments with a varying molecular fraction. However, we caution that the data obtained via reduced-potential scaling are approximate, but are reasonable estimates until explicit calculations become available.

\section{Conclusion}

Rate coefficients for the deexcitation of rotational excited HF due to He collisions were computed using the close-coupling method and an accurate potential energy surface. The adopted ab initio PES agrees well with an experimentally deduced empirical PES. Differences of $75 \%$ and larger were found with previous HF-He scattering calculations that used a less reliable PES. New rate coefficients were obtained for HF rotational levels $j=1-20$ for $0.1-3000 \mathrm{~K}$ due to He. A recently introduced scaling approach was used to estimate rate coefficients for HF-H and missing $\mathrm{HF}-\mathrm{H}_{2}$ collisional data.

Acknowledgements. Work at UGA was supported by NASA grant NNX12AF42G, at Penn State by NSF Grant No. PHY-1203228, and at UNLV by NSF Grant No. PHY-1205838. We thank Ad van der Avoird and T. Stoecklin for sending their potential subroutines and for helpful correspondence.

\section{References}

Agúndez, M., Cernicharo, J., Waters, L. B. F. M., et al. 2011, A\&A, 533, L6 Alexander, M. H., \& Manolopoulos, D. E., 1987, J. Chem. Phys., 86, 2044

Arthurs, A. M., \& Dalgarno, A. 1963, Proc. Roy. Soc. A, 256, 540

Brunet, S. M. K., Guo, J. Z., Carrington, T., Filseth, S. V., \& Sadowski, C. M. 2002, J. Chem. Phys., 116, 3617

Chapman, W. B., Weida, M. J., \& Nesbitt, D. J. 1997, J. Chem. Phys., 106, 2248

Coxon, J. A., \& Hajigeorgiou, P. G. 1990, J. Mol. Spectr., 142, 254

Dubernet, M. -L., Alexander, M. H., Ba, Y. A., et al. 2013, A\&A, 553, A50

Fajin, J. L. C., Fernandez, B., Mikosz, A., \& Farrelly, D. 2006, Mol. Phys., 104, 1413

Faure, A., \& Lique, F. 2012, MNRAS, 425, 740

Faure, A., Valiron, P., Wernli, M., et al. 2005, J. Chem. Phys., 122, 221102

Flower, D. R. 2007, Molecular Collisions in the Interstellar Medium, 2nd edn. (Cambridge: Cambridge Univ. Press)

Guillon, G., \& Stoecklin, T. 2012, MNRAS, 420, 579

Guillon, G., Stoecklin, T., Voronin, A., \& Halvick, Ph. 2008, J. Chem. Phys., 129,104308

Hutson, J. M., \& Green, S., 1994, MOLSCAT Computer Code, version 14 Collaborative Computational Project No. 6 of the Engineering and Physical Sciences Research Council (UK)

Irikura, K. K. 2007, J. Phys. Chem. Ref. Data, 36, 389

Kalugina, Y., Lique, F., \& Marinakis, S. 2014, Phys. Chem. Chem. Phys., 16, 13500

Lovejoy, C. M., \& Nesbitt, D. J. 1990, J. Chem. Phys., 93, 5387

Monje, R. R., Phillips, T. G., Peng, G., et al. 2011, ApJ, 742, L21

Monje, R. R., Lord, S., Falgarone, E., et al. 2014, ApJ, 785, 22

Moszynski, R., Wormer, P. E. S., Jeziorski, B., \& van der Avoird, A. 1994, J. Chem. Phys., 101, 2811

Moszynski, R., de Weerd, F., Groenenboom, G. C., \& van der Avoird, A. 1996, Chem. Phys. Lett., 263, 107

Neufeld, D. A., Zmuidzinas, J., Schilke, P., \& Phillips, T. G. 1997, ApJ, 488, L141

Neufeld, D. A., Sonnentrucker, P., Phillips, T. G., et al. 2010 A\&A, 518, L108

Phillips, T. G., Bergin, E. A., Lis, D. C., et al. 2010, A\&A, 518, L109

Reese, C., Stoecklin, T., Voronin, A., \& Rayez, J. C. 2005, A\&A, 430, 1139

Rodwell, W. R., Sin Fai Lam, L. T., \& Watts, R. O. 1981, Mol. Phys., 44, 225

Roueff, E., \& Lique, F. 2013, Chem. Rev., 113, 8906

Schöier, F. L., van der Tak, F. F. S., van Dishoeck, E. F., \& Black, J. H. 2005, A\&A, 432, 369

Stark, K., \& Werner, H.-J. 1996, J. Chem. Phys., 104, 6515

Stoecklin, T., Voronin, A., \& Rayez, J. C. 2003, Chem. Phys., 294, 117

Tizniti, M., Le Picard, S. D., Lique, F., et al. 2014, Nat. Chem., 6, 141

van der Tak, F. 2012, Adv. Space Res., 49, 1395

van der Tak, F. F. S., Ossenkopf, V., Nagy, Z., et al. 2012, A\&A, 537, L10

Walker, K. M., Yang, B. H., Stancil, P. C., Balakrishnan, N., \& Forrey, R. C. 2014, ApJ, 790, 96

Wiesenfeld, L., \& Faure, A. 2013, MNRAS, 432, 2573

Wigner, E. P. 1948, Phys. Rev., 73, 1002

Yang, B. H., Stancil, P. C., Balakrishnan, N., Forrey, R. C., \& Bowman, J. M. 2013, ApJ, 771, 49

Zhang, D. H., \& Zhang, J. Z. H. 1993, J. Chem. Phys., 99, 6624 